\title{
El arte en un acto \\ sensible: el niño en \\ procesos creativos
}

\section{Artículo de reflexión}

\section{Sandra Yaneth Chaparro Cardozo}

Fundación Universitaria Juan de Castellanos, Colombia sandrayanethchaparro@gmail.com

\section{Elver Chaparro Cardozo}

Fundación Universitaria Juan de Castellanos, Colombia eprieto@jdc.edu.co

\section{Edixon Alberto Prieto Acevedo}

Fundación Universitaria Juan de Castellanos, Colombia echaparro@jdc.edu.co

Cómo citar este artículo: Chaparro Cardoso, Sandra; Chaparro Cardoso, Elver; Prieto Acevedo Edixon (2018). El arte en un acto sensible: el niño en procesos creativos. Calle14: revista de investigación en el campo del arte, 13 (23) pp. 186-196. DOI: https://doi. org/10.14483/21450706.12998 


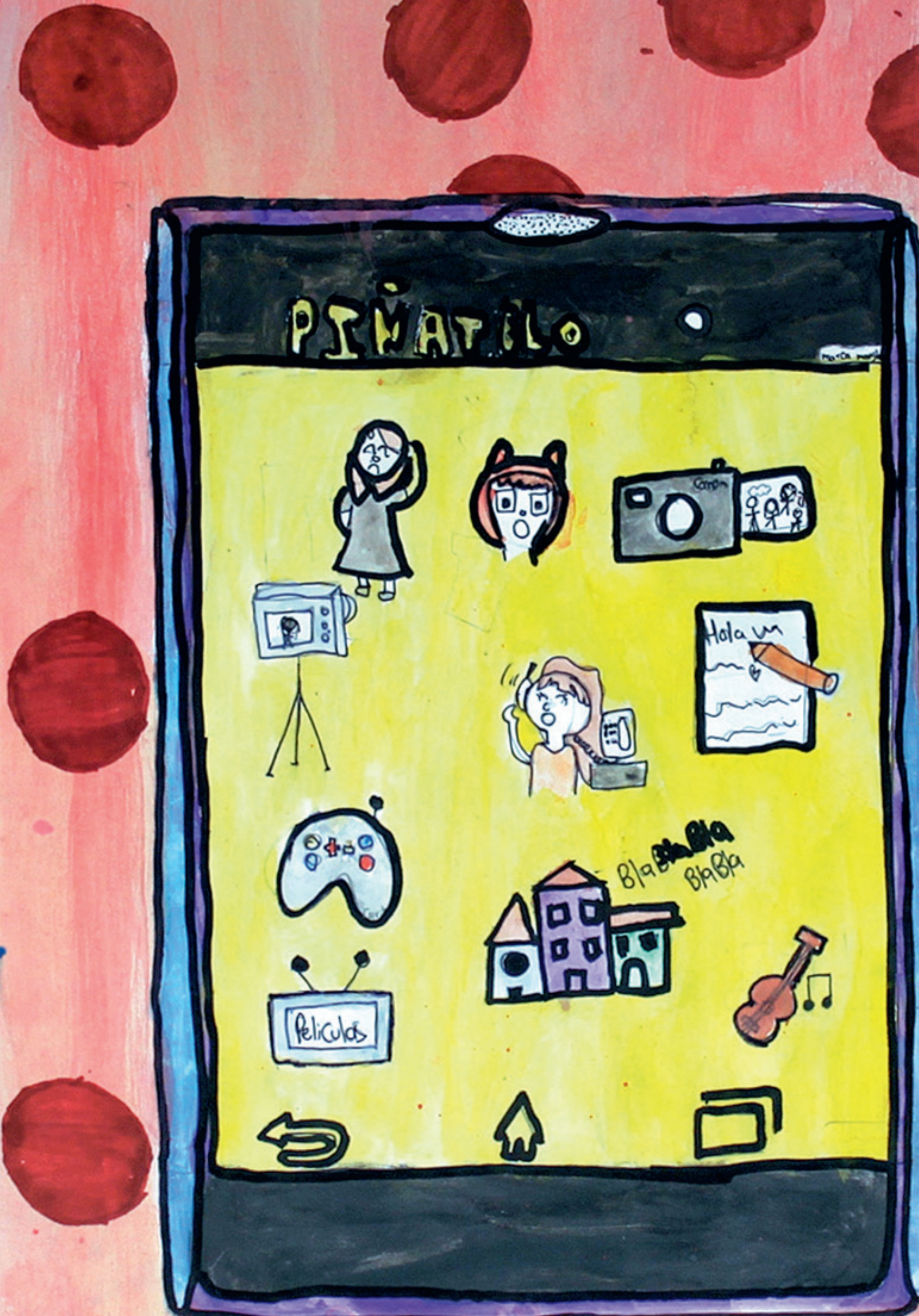




\title{
Resumen
}

El presente artículo indaga entorno a los procesos artísticos y su incidencia en la formación de la creatividad, y surge como parte del proyecto Escuela y Creatividad: Estrategias y Fomento de la Facultad de Ciencias de la Educación y Humanidades de la Fundación Universitaria Juan de Castellanos de Tunja, Boyacá. En este estudio se revisaron algunas relaciones entre el arte y la creatividad, partiendo de la observación e interpretación de las intervenciones pictóricas de los niños. Lo que conllevó al siguiente interrogante: ¿Qué tipo de procesos artísticos se desarrollan en la infancia y cómo se articulan con los procesos creativos? Esta investigación se orientó a partir del estudio de casos y tiene por objetivo determinar prácticas artísticas que se desarrollan en la infancia y su articulación en el desarrollo de procesos creativos en niños y niñas. De igual forma se realizó un reconocimiento inicial de la concepción de las artes y la plástica frente a la creatividad, y de la capacidad creadora y artística en la infancia.

\section{Palabras claves}

Prácticas artísticas, arte, creatividad, infancia

Art in a sensitive act: the child in creative processes

\begin{abstract}
This article investigates the artistic processes and their impact on the formation of creativity. It is part of the project School and Creativity: Strategies and Promotion of the Faculty of Education Sciences and Humanities of the University Foundation Juan de Castellanos of Tunja. In this study, some relationships between art and creativity were reviewed, based on the observation and interpretation of pictorial interventions by children. This led to the following question: What kind of artistic processes are developed in childhood and how are they articulated with creative processes? This research was guided from the case studies and aims to determine artistic practices that are developed in childhood and their articulation in the development of creative processes in children. In the same way, an initial recognition of the conception of the arts facing creativity, and of the creative and artistic capacity childhood was carried out.
\end{abstract}

\section{Keywords}

Artistic practices, art, creativity, childhood.

\section{L'art dans un acte sensible : l'enfant dans les processus créatifs}

\section{Résumé}

Cet article étudie les processus artistiques et leur impact sur la formation de la créativité. II fait partie du projet École et Créativité : Stratégies et Promotion de la Faculté des Sciences de l'Education et des Sciences Humaines de la Fondation Universitaire Juan de Castellanos de Tunja. Dans cette étude, quelques relations entre l'art et la créativité ont été passées en revue, basées sur l'observation et l'interprétation d'interventions picturales par des enfants. Cela a conduit à la question suivante: Quels types de processus artistiques sont développés dans l'enfance et comment s'articulent-ils avec des processus créatifs ? Cette recherche a été guidée à partir des études de cas et vise à déterminer les pratiques artistiques qui se développent dans l'enfance et leur articulation dans le développement des processus créatifs chez les enfants. De même, une première reconnaissance de la conception des arts face à la créativité, et de la capacité créative et artistique de l'enfance a été réalisée.

\section{Mots clés}

Pratiques artistiques, art, créativité, enfance. 


\section{Resumo}

Este artigo investiga os processos artísticos e seu impacto na formação da criatividade. Faz parte do projeto Escola e Criatividade: Estratégias e Promoção da Faculdade de Ciências da Educação e Humanidades da Fundação Universitária Juan de Castellanos de Tunja. Neste estudo, foram analisadas algumas relações entre arte e criatividade, com base na observação e interpretação das intervenções pictóricas por parte das crianças. Isso levou à seguinte questão: que tipo de processos artísticos são desenvolvidos na infância e como eles são articulados com processos criativos? Esta pesquisa foi orientada a partir dos estudos de caso e visa determinar práticas artísticas que são desenvolvidas na infância e sua articulação no desenvolvimento de processos criativos em crianças. Do mesmo modo, realizou-se um reconhecimento inicial da concepção das artes voltadas para a criatividade e da criatividade artística criativa.

\section{Palavras-chave}

Práticas artísticas, arte, criatividade, infância.

\section{Imasami sug iura pangapi ruraskapi suma sintiskasina uchuia imasami pai iachaiku rurangapa}

\section{Maillallachiska:}

Kai kilkaskapi tapumi imasami iachachirka kai iachai tasa kai wasi iachachidirupi rurariskami sug iachachi atun wasipi atun iachakunawa kai wagki suti Juan Castellano kai Tunjamanda i Boyacamanda. Kai iachaikuipi kawarirkami chasa tsabajukuna ruraikuna i sugrigcha kawanaikui ruraska sug uchuia wambrakuna. Kai chasa pusurkami sug tapuima ¿ imasata iurarirka kai uchullakunata ruraikuna i imasami Tukui iachachima kagta? Kai tapuikuna rurarikami kaiarispa iachachi sug iachachiskakunamandaitata i imasami uchullakuna rurarkakuna chasa kai sumami kawachirirka iapa suma kagta chi ruraikuna kai kariwambrakuna i warmiwambrakuna.

\section{Rimangapa Ministidukuna:}

Imasami iachaikurka rural kilkangapa, llunchii, ruraikuna uchullakuna. 


\section{Introducción}

En esta propuesta se buscó indagar las posibilidades que tienen las prácticas artísticas y su incidencia en la formación de la creatividad. En un primer acercamiento que se realizó desde del proyecto Escuela y creatividad: estrategias y fomento de la Facultad de Ciencias de la Educación y Humanidades de la Fundación Universitaria Juan de Castellanos se indagó en las relaciones entre creatividad y arte.

Dado que se reconoce el papel de la educación artística en la primera infancia surge la preocupación por estudiar su incidencia en los procesos creativos de los niños y las niñas, en este caso con procesos pictóricos. La inserción de la educación artística en la escuela ha permitido fomentar diferentes miradas del arte, y de esta manera, observar cómo se está formando la creatividad en la primera infancia desde una dimensión artística. Esto también arroja una mirada sobre los juicios valorativos que el niño y la niña asignan a sus creaciones. Para ello se propuso examinar algunas formas de creatividad y su relación con las prácticas artísticas en la primera infancia. En el estudio surgieron interrogantes tales como: ¿Cómo se está desarrollando la creatividad en los procesos artísticos en los niños? ¿De qué manera el arte influye en el aprendizaje?

De esta manera, se planteó el problema: ¿Qué tipo de prácticas artísticas se desarrollan en la primera infancia y cuál es su articulación en los procesos creativos? Para ello se propuso desarrollar un análisis de material relacionado con las prácticas artísticas desarrolladas por los niños/niñas en la primera infancia, junto con el análisis de conceptos sobre infancia, arte y creatividad, y abordar las múltiples miradas desde lo artístico y lo creativo que se desarrollan en la escuela.

Este estudio permitió justificar y explicar trabajos relacionados con la creatividad, y de esta manera detectar los elementos de la creación pictórica. Esta propuesta investigativa nace a partir de profundizar y ofrecer mejores orientaciones sobre el papel que tiene el arte en la educación.

La metodología de este estudio es de corte cualitativodescriptivo, con la cual se realizó un reconocimiento de manifestaciones artísticas como pintura y dibujo, entre otras, realizando un análisis preliminar sobre su articulación con los procesos creativos y las producciones artísticas de niños y niñas.
Se tomaron algunos estudios de Viktor Lowenfeld (1961) sobre el desarrollo de la capacidad creadora como parte de las didácticas en la educación artística, teniendo en cuenta que no es desconocida la capacidad creadora en la primera infancia. Por ello se plantea una relación de las prácticas artísticas y el desarrollo de la creatividad mediante la creación de propuestas de los niños/as, en las cuales se observe esa estrecha relación que existe entre las prácticas artísticas en comunidades escolares y su influencia en procesos artísticos.

\section{Metodología}

Teniendo en cuenta los procesos creativos mediados por las manifestaciones artísticas, la investigación cualitativa permite abordar las formas de entender y conocer cómo se traducen los lenguajes artísticos y los procesos creativos en la primera infancia dentro de una comunidad escolar o fuera de esta.

El enfoque cualitativo se orienta más a un esfuerzo por comprender la realidad social en que los niños determinan sus procesos artísticos y su articulación a los procesos creativos. En primer lugar, se realizó un acercamiento analítico para identificar cómo se están llevando las prácticas artísticas de los niños/as que están en proceso de formación. En segundo lugar, hay una observación que se encuentra en proceso, la cual se desarrollara mediante la recolección de experiencias y la indagación teórica sobre los procesos creativos y su relación con las prácticas artísticas.

La propuesta implica el trabajo de exploración y formación sobre cómo concebir el arte, además de las formas de apropiación de una educación en la práctica artística que promueva el estudio de la infancia y la innovación en los lenguajes particulares de los niños/niñas.

\section{Discusión}

El arte infantil visto desde perspectivas de la expresión se podría definir como un estado de lo imaginario, como una estructura estratégica en la producción y reproducción de imágenes. A partir de esto se podría plantear el siguiente cuestionamiento: ¿los niños desarrollan un pensamiento a través del arte? Teniendo esta pregunta, se crea un problema y se configura un objeto de estudio sobre la investigación en el arte infantil. Esta investigación se estructura dentro de los procesos de pensamiento en la infancia y aspira a ser un aspecto 


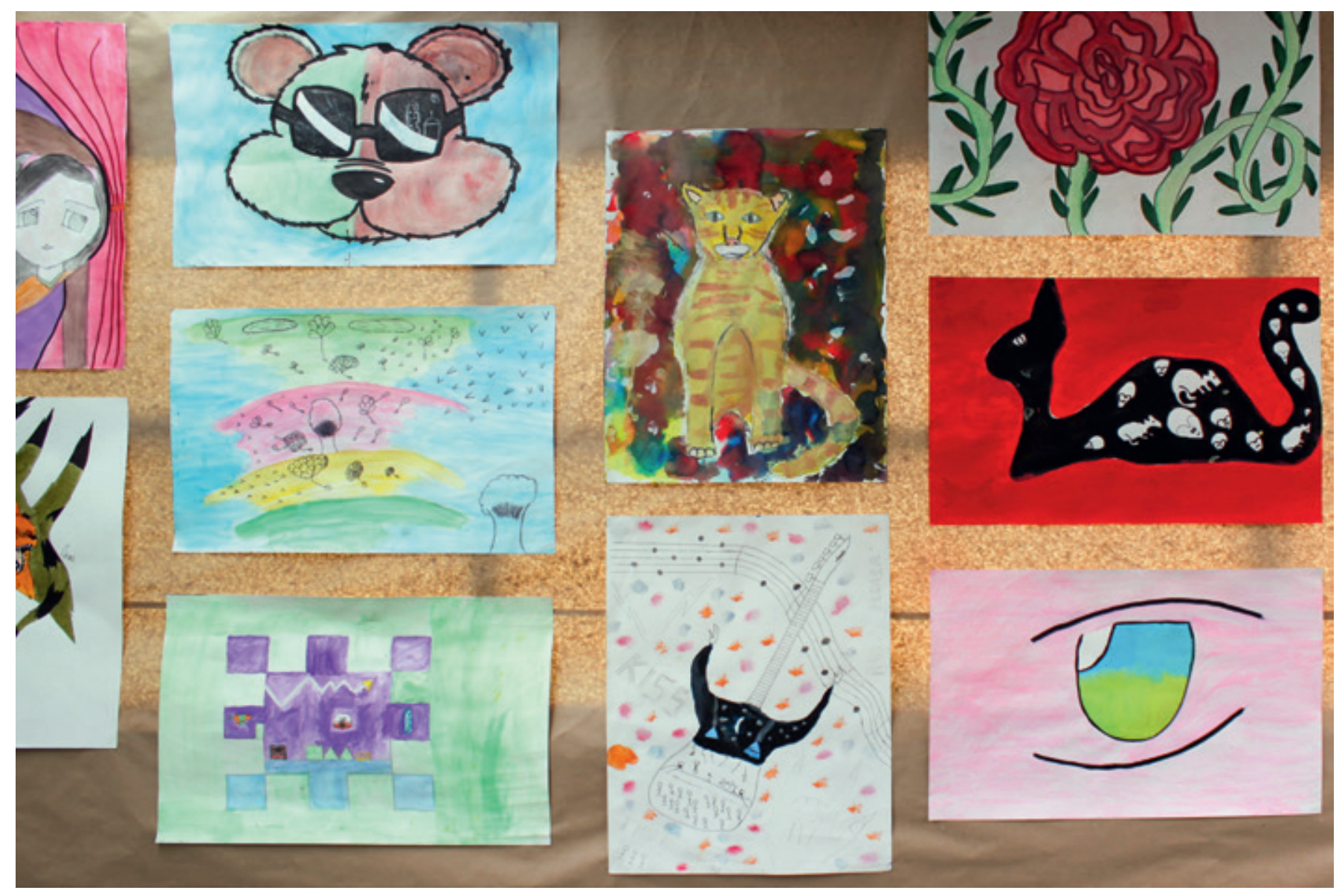

Trabajo de artes plásticas de estudiantes nivel 11 Centro Educativo Libertad, CEL. 2015.

fundamental para mejorar los procesos de creatividad de los niños y niñas. El desarrollo y difusión de nuevas teorías y modos de organizar el aprendizaje artístico se pueden rastrear a partir de la década de 1970, donde se plantea que la concepción de la infancia experimenta nuevos cambios, superando aquella visión del niñoobjeto señalada por Franco Frabboni (1986). (Sarlé y Hernández, 2014: 15)

En contraste, se hallan numerosos investigadores con propuestas que con gran éxito han sido aplicada en varios países. Tales propuestas han crecido en todos los sentidos, con diversas adaptaciones de acuerdo a los contextos sociales y culturales de cada niño. Con lo anterior, se busca reforzar la idea de potenciar la creación artística desde la cultura del niño y la niña, creando un dialogo entre aprender, disfrutar, preguntar y sorprenderse por su espontaneidad creadora. Sobre esto:

Gardner rompe con el esquema de la inteligencia dándole al concepto un nuevo significado al referirse con él a una amplia variedad de capacidades humanas. Congruente con esto, casi todos podemos reconocer la existencia de la creatividad en la música y en las plásticas (...).

(Macias, 2002: 31)

Por ello, los procesos de enseñanza y aprendizaje permiten acercar a los niños y niñas a otras formas de expresión, estimulando la imaginación, la creatividad como parte de la identidad crítica individual. En este orden de ideas, el dibujo infantil nace espontáneamente, y buena parte de la investigación comienza sobre las características propias de la primera infancia o la niñez. Muchos autores han dedicado diversos estudios a la expresión infantil, como es el caso de Howard Gardner y Víctor Lowenfeld.

El arte, como herramienta en el estudio de la imagen infantil, es visto como parte de los procesos creativos, donde forma parte de un ejercicio de creación que parte de las destrezas sensible del niño, contribuyendo a la expresión del pensamiento infantil, el desarrollo de las capacidades de percepción, de observación y de comunicación, en un espacio donde niños y niñas pueden hablar de sus sentimientos, inquietudes, emociones 
y temores, y pueden conocerse frente al mundo desde sus pinturas u objetos de creación, así como frente a los posibles espectadores.

La creación de espacios en los que el niño y la niña puedan narrar sus procesos personales de forma anecdótica, haciendo uso de diferentes materiales, posibilita el desarrollo de habilidades manuales y cognitivas. Con esto se busca reforzar una actitud mental en la que se supere y tome posición crítica frente a los comentarios del adulto y pueda transformarse el desarrollo la capacidad creadora. A esto se refiere Viktor Lowenfeld hablando del desarrollo del arte infantil en la escuela (1973): el dibujo de los niños tiene unas características de acuerdo a su etapa, y de esta manera se expresan espontáneamente de acuerdo a su nivel de desarrollo. Sobre este aspecto Puleo plantea:

Tomando en consideración que los estudios sobre las características del dibujo infantil realizados por autores reconocidos tales como Lowenfeld (1961), Luquet (1927), Harris (1963) y Piaget (1975), etc., han sido llevados a cabo fuera de nuestro contexto social venezolano y varias décadas atrás, se plantea la necesidad de indagar las características evolutivas del dibujo en el niño(a) de 1 a 6 años de edad, en el contexto sociocultural merideño actual, tomando en cuenta que en el presente, el niño(a) recibe una mayor y más temprana estimulación visual por parte de sus padres, los medios de comunicación de masas y la escuela. (Puleo, 2012: 158)

Según lo anterior, el concepto de arte infantil aparece como parte de una unidad creativa que se transforma en color. Con ello se busca descubrir y establecer vínculos emocionales con las realidades de los pequeños artistas, tomando como referente plástico al pintor Henry Matisse (1869), quien considera la pintura infantil como expresionista. Es así que el niño usa el color sin ninguna intención representativa o simbólica de sus sentimientos. Los niños pintan con el fin de disfrutar y salirse un poco de su realidad, o talvez de generar narraciones mediante los colores y las formas. Por lo tanto, se hace necesario conocer los procesos de creatividad en los que el niño descubre y experimenta diferentes herramientas, texturas y materiales. De esta manera, el niño descubre su universo en las acciones motrices y en el acto de jugar a crear pintando. En línea con esto,

La creatividad ha existido desde siempre, es una habilidad del ser humano y, por lo tanto, vinculada a su propia naturaleza. Sin embargo, por mucho tiempo, la creatividad como concepto fue un tema no abordado y por lo mismo poco estudiado, es hasta años recientes donde surgen teóricos que se abocan a profundizar sobre el tema y se desarrollan trabajos y aportaciones alusivas a este concepto. (Esquivas, 2004: 3)

En este sentido, el arte es un medio de expresión y experimentación, ya sea desde la pintura, el dibujo, la música, el teatro o la danza. Entender el universo mediante las manifestaciones gráficas y, a la vez, cómo estas intervienen en los procesos creativos, que, para este caso, son procesos pictóricos en los que el niño hace una reconstrucción del universo de forma distinta y de esta forma expresa su realidad, y donde el "estilo creativo de una persona parte del modo en el cual utiliza la información para estimular su creatividad. Cada estilo creativo prefiere un método distinto para generar y evaluar ideas". (Valquí, 2009: 8)

Por ello, los universos que construyen los niños desde su pensamiento creativo son expresados de diferentes manaras. Viktor Lowenfeld (1984) relaciona esto con el desarrollo del arte infantil, asignando nueve etapas al desarrollo cognitivo: 1) Etapa del garabato (2 a los 4 años), la representación intuitiva de los objetos; 2) Etapa del garabateo desordenado: el niño comienza a garabatear en torno a los 18 meses; 3) Etapa del garabateo controlado: el niño se va dando cuenta de las líneas que traza y de la afectación que tienen en el papel; 4) Etapa del garabateo con nombre: el niño busca dar significado a los garabatos; 5) Etapa preesquemática (4 a los 7 años): el niño reconoce su acción dedicándole un mayor tiempo de trabajo; 6) Etapa esquemática (7 a los 9 años): el niño genera una semejanza con la realidad; 7) Etapa del realismo (9 a los 12 años): representación del dibujo en líneas en las cuales le da un sentido a la imagen; 8) Etapa del pseudonaturalismo (12 a los 13 años): los dibujos tienen mayor detalle y se observa mayor detalle sexual; 9) Etapa de la decisión (13 a los 14 años): hay una búsqueda de técnicas artísticas y pulimento de las imágenes.

Teniendo en cuenta las edades de los niñas y niños, varían sus intereses en la elaboración y atracción por la elaboración de los dibujos. Para ello se requiere del uso de diferentes materiales y de espacios que permitan potenciar la imaginación y la creatividad. En este proceso artístico incide la creatividad y la libre expresión. Seguimos a Jean Jacques Rousseau, quien manifestaba que el niño debía identificar sus errores por sí mismo, sin la presión de un adulto haciéndole ver 
sus dificultades, lo cual permite el desarrollo y fortalecimiento de la autocrítica. Así, el arte es un camino al auto-reconocimiento: en la construcción de dibujos que buscan narrar o contar una realidad, el niño y la niña van revelando sus pensamientos con plena libertad.

Por ello, (...) las personas creativas encuentran en el entorno cultural que les rodea estímulos de todo tipo para el desarrollo de pensamientos originales y enriquecen el inventario de soluciones creativas que han ayudado a dar más calidad de vida a la sociedad. Un bagaje más rico de soluciones ingeniosas en todos los ámbitos contribuye, por ósmosis, a que el sistema educativo sea más efectivo en la transmisión de la imaginación y la inventiva, con lo cual se desarrollará con mayor potencia la creatividad de los alumnos, cerrándose así el triángulo virtuoso de crecimiento del país en creatividad e innovación. (Guilera, 2011: 26)

Por otra parte, hay artistas que piensan que las artes son tan solo una práctica o habilidad para la creación en la que actúan emociones, represiones, y que permite poner de manifiesto la experiencia del juego de colores. En este sentido, los niños y las niñas exploran texturas y formas que les permiten tener experiencias sensoriales. Esta experiencia no solo potencia la imaginación y la creatividad, sino que fomenta la seguridad dado que el dibujo se caracteriza por tener una variedad de matices en sus formas, líneas, trazos y colores, lo que le permite al niño potenciar su habilidad expresiva innata.

\section{En camino al arte infantil, signos de pensamiento creativo en la escuela}

En la primera mitad del siglo XIX los primeros manuales y cartillas escolares de dibujo para la infancia fueron elaborados por pedagogos como Johann Heinrich Pestalozzi (1746-1872) con su libro El ABC de la intuición o la intuición de las proporciones, y Friedrich Froebel (1782-1852), quien contribuyó con su proyecto curricular en jardines de infancia organizados en torno al principio del juego. Por otra parte, Viktor Lowenfeld, en el siglo XX, suscitó un cambio de los esquemas entre el modo tradicional de enseñanza del dibujo en la escuela y su nueva concepción de la educación artística como desarrollo de la creatividad.

Ahora bien, cuando se trata de la "enseñabilidad" del arte o de las prácticas artísticas en la escuela, el maestro de la disciplina busca potenciar las habilidades

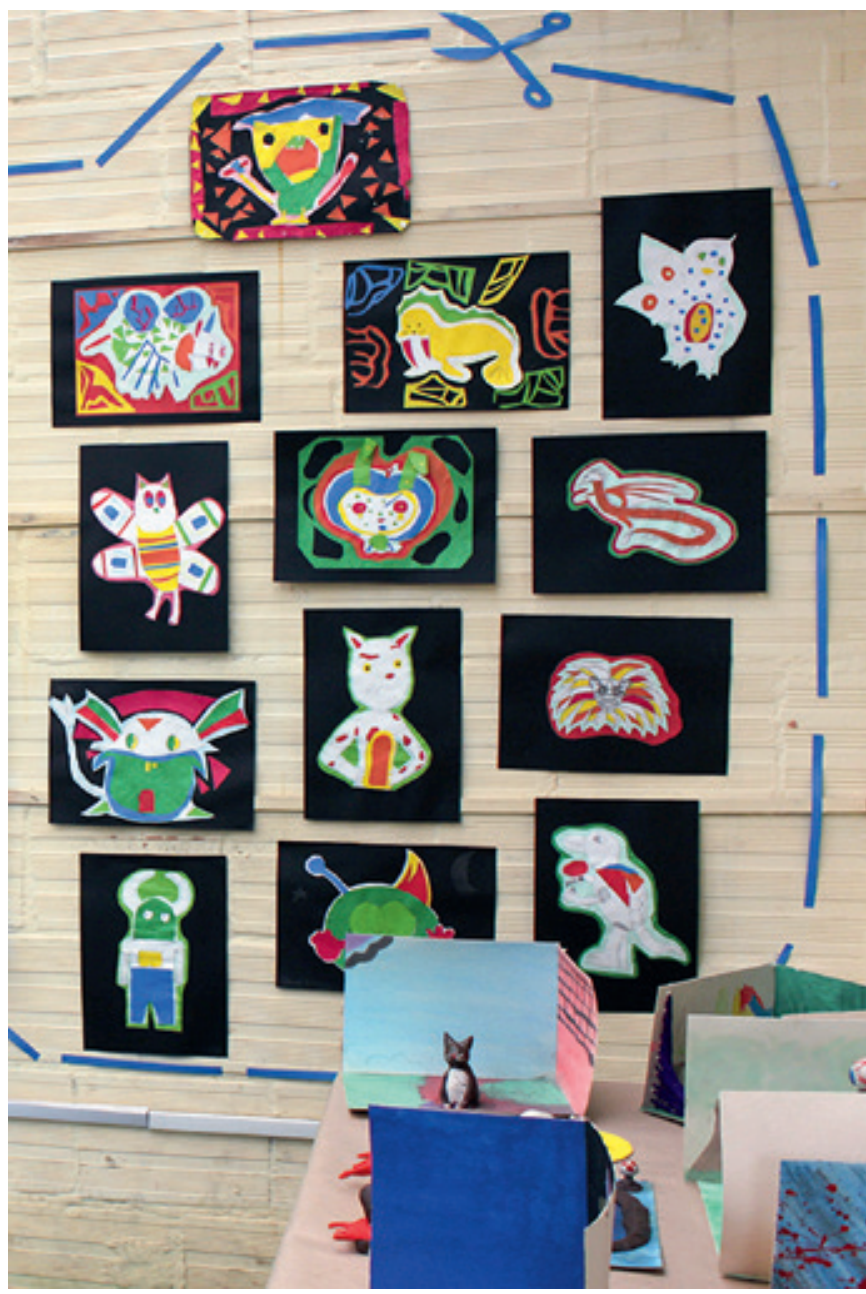

Trabajo de artes plásticas de estudiantes del Centro Educativo Libertad, CEL. 2015.

de los niños en el manejo del color, la forma, el estilo, o simplemente el mensaje que este quiere transmitir. Frente a esto, pintores como Picasso coinciden con estudiosos como Gradner (1993: 180), quien anota que "algunos describen el cubismo como un intento por dibujar como lo hacen los niños y de ver del mismo modo que ellos, o como una vuelta (posiblemente inconsciente) a los dibujos garabateados, experimentos y dibujos marginales".

Estos son intentos del niño por expresar sus sentimientos, en los que no solo se trata de líneas, trazos, formas y detalles, sino de una historia que aspira ser dibujada. Estas formas tan particulares de expresión son las que se pueden denominar como creativas. En ellas el niño potencia una actitud que busca ser reconocida por los adultos; en otras palabras, quiere ser animado a continuar creando, imaginado y maravillándose sobre su actividad artística. 


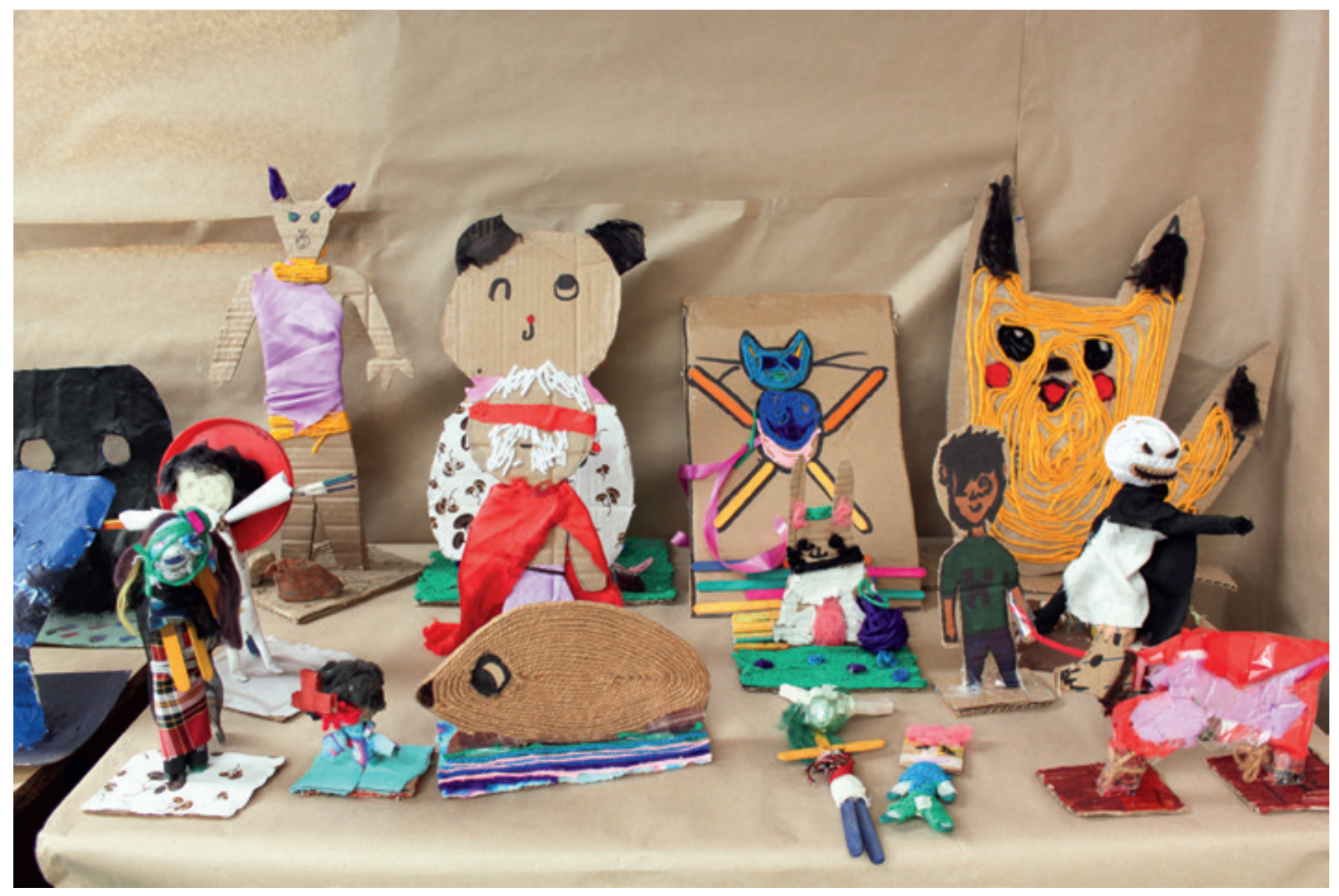

Trabajo de artes plásticas de estudiantes del Centro Educativo Libertad, CEL. 2015.

Con esto el arte infantil, visto desde la comunicación y los lenguajes de creación, es parte fundamental de los fenómenos que intervienen en los procesos creativos. Por ello, es fundamental reconocer la importancia de la capacidad creadora en la primera infancia dentro del mundo de lo pictórico, tal como en su momento Vasili Kandinsky, artista de vanguardia, reconoció la importancia de la producción artística de los niños y niñas.

El estudio de la actitud del niño en sus distintas etapas (cuando dibuja, pinta, pega o modela) permite entender cómo percibe lo que está exteriorizando y cómo el guía la diferenciación de cada actividad. Su cuerpo, como vehículo de su mente y de sus emociones, deja ver un proceso de memoria visual: el niño está en constante juego con los matices o la textura de un color, transpone formas o manipula volúmenes en el espacio de forma inconsciente y no muy premeditada.

Ciertamente, a nivel histórico, se encuentran datos sobre la enseñanza del dibujo y la manera como se implantó en el currículo escolar, al mismo tiempo que se organizaron y generalizaron los sistemas educativos durante la primera mitad del siglo XIX. Por ejemplo, aparece el interés por crear orientaciones de educación artística en la escuela a partir de la obra de grandes filósofos como Platón y Aristóteles, quienes afirmaron la importancia de la enseñanza del dibujo en los niños.

Al mismo tiempo, el sistema educativo comenzó a incluir el dibujo dentro de las materias obligatorias del currículo, tanto para la escuela primaria como para la secundaria. Esto vino con la necesidad de orientar las artes académicas y formar al profesorado en esta materia. Vale la pena resaltar que la anterior implementación se produjo en un contexto histórico y social caracterizado por la industrialización en lo productivo, y la revolución francesa y la independencia norteamericana en lo político.

Luego de este primer acercamiento al arte y a su incidencia en la creatividad, se trata de hallar las estrategias que los maestros pueden usar para potenciar las habilidades artísticas de niñas y niños. Frente a estos, es importante comprender las dinámicas institucionales en las que se promueve a las Escuelas Creativas de Ken Robinson (2015). Este autor indica que las 
escuelas pasan por momentos difíciles para los niños y los maestros; estos últimos sufren una presión enorme en el desarrollo de habilidades y competencias sobre un determinado saber. Estos elementos represivos de la escuela cohíben tanto al niño como al maestro en la posibilidad de imaginar, reinventar y crear, así como en la posibilidad de generar soluciones prácticas y derrumbar ideologías institucionales de los sistemas educativos actuales.

Hay que tener en cuenta que las actividades artísticas están incluidas como competencias escolares en los estándares educativos del Ministerio de Educación Nacional ${ }^{1}$ de Colombia y buscan una orientación pedagógica para la educación artística que promueva la cultura y el reconocimiento a la diversidad de las expresiones, así como el fortalecimiento de las capacidades de participación de niñas y niñas en las escuelas. El aumento reciente en los proyectos conducidos por artistas y educadores sugiere que el clima para la actividad investigativa en las artes en infancia está cambiando, no solo en los países desarrollados, sino también en Latinoamérica y demás países en desarrollo como Colombia.

En la comunidad académica se están estudiando las problemáticas relacionadas con el arte y su incidencia en la actividad creativa. Esto se puede observar en las memorias, artículos, trabajos de investigación y opiniones de artistas, que son publicadas en medios poco valorados y muy marginados. Por lo tanto, las finalidades y principios pedagógicos del proyecto educativo institucional deben constituirse en un marco desde el cual lo artístico adquiere sentido educativo.

De ahí en adelante, la manera como el conocimiento artístico se transforma en saber artístico de la comunidad educativa es resultado de las maneras específicas del hacer educativo que asume lo artístico y las didácticas, integrando las manifestaciones del arte, sin que por ello la educación asuma la total responsabilidad de formar artistas. El significado de las actividades

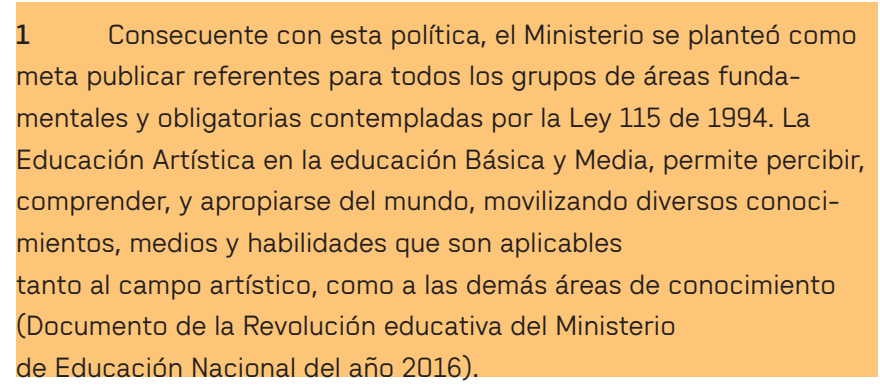

artísticas ha mostrado que estas promueven el desarrollo de la creatividad gráfica, verbal y perceptiva.

Las artes y el acto creador responden a productos artísticos elaborados por el ser humano y que nacen de los procesos imaginativos, fantásticos e innovadores. Estos procesos creativos se ven presentes en el desarrollo cognitivo y potencializarían la fluidez verbal, la originalidad verbal y gráfica, la flexibilidad y la elaboración, en términos de autores como Torrance (1978).

\section{Conclusiones}

Desde esta perspectiva es evidente que lo artístico, como propuesta educativa escolar, no se sustrae a la responsabilidad sobre los fines específicos y generales a los cuales apunta un proceso educativo. El arte constituye un componente más de la estructura curricular mediante la cual se quieren alcanzar, de forma concreta, las finalidades del proyecto educativo institucional.

Los garabatos que ejecuta el niño se traducen en ideas, y en cada nuevo intento por manifestar sus imágenes está la realidad como la percibe. Pintar significa proponerles a los niños una experiencia donde jugarán a crear sus propios colores. Desde la mancha, el niño descubrirá en su juego de crear una materialización de su universo.

El niño busca desarrollar una concepción global de la forma en conjunto con la mancha, lo que determinará, de acuerdo con la edad, la estructura y los detalles. El sugerir, en el acto de dibujar, la concentración y rigurosidad, en lo que el adulto o el niño pueden considerar como autentico, es un tanto relativo para el segundo. Cuando el niño y la niña pintan, se emocionan ante todo con el color y la forma, con lo que aparece en el papel o en la pared.

El niño se siente atraído por los elementos para pegar; centra su atención en la transposición, en la descomposición y composición de los figurines que él construye o modela en plastilina o arcilla. Es por esto que el niño hace del arte una sorpresa y la comparte, haciendo participe, a los que se hallan a su alrededor, de sus emociones de lo posiblemente bello; le encanta establecer un contacto directo con el público.

Finalmente, la conciencia que tienen el niño y la niña de la transformación de su arte vuelve a su hacer algo 
más que actividades manuales, son comportamientos creativos que además de transformar un elemento cuantas veces se desee, permiten recrear o dar vida a una concepción mental de lo bello.

\section{Referencias}

Esquivas, S. M. (2004). Creatividad: definiciones, antecedentes y aportaciones. Digital Universitaria, 2-17.

Garnerd, H. (1993). Mentes creativas. España: Ediciones Paidós.

Guilera, L. A. (2011). Anatomía de la creatividad. Estados Unidos: FUNDIT.

Macías, M. A. (2002). Las inteligencias múltiples.

Universidad de Norte, 27-38.

Puleo, R. E. (2012). La evolución del dibujo infantil, una mirada desde el contexto sociocultural. Educares, 157-170.

Sarlé, P., Elizabeth, I., y L. Hernández. (2014). Arte, educación y primera infancia: sentidos y experiencias. España: OIE.

Robinson, Kent. (2015) Escuelas creativas. Edito Grijalbo.

Valquí, V. R. (2009). La creatividad: conceptos. Métodos y aplicaciones. Iberoamericana de Educación, 1-11. 
